\title{
Noninvasive evaluation of ovarian endometriosis: a single-center experience
}

\author{
Kun Zhao ${ }^{1,2}$, Pengpeng $Q u^{2}$ \\ ${ }^{1}$ Tianjin Medical University, Tianjin, China; ${ }^{2}$ Department of Gynecological Oncology, Tianjin Central Hospital of Gynecology and Obstetrics, \\ Tianjin, China \\ Contributions: (I) Conception and design: K Zhao; (II) Administrative support: P Qu; (III) Provision of study materials or patients: K Zhao; (IV) \\ Collection and assembly of data: K Zhao; (V) Data analysis and interpretation: K Zhao; (VI) Manuscript writing: All authors; (VII) Final approval of \\ manuscript: All authors. \\ Correspondence to: Pengpeng Qu. Department of Gynecological Oncology, Tianjin Central Hospital of Gynecology and Obstetrics, Tianjin, China. \\ Email: qu.pengpeng@hotmail.com.
}

Background To investigate the relationships of cancer antigen (CA) 125, CA 19-9, prothrombin time (PT), activated partial thromboplastin time (aPTT), thrombin time (TT), fibrinogen (FIB), and D-dimer values with ovarian endometriosis (OEM), and to explore the validation of biomarkers for noninvasive preoperative evaluation of patients with severe OEM.

Methods: This retrospective case-control study included 413 women with OEM (of whom 143 cases were stage I to II, 139 cases were stage III, and 131 cases were stage IV, respectively) and 158 women without OEM as controls. Subjects' serum CA-125 and CA19-9 levels, and coagulation test results (serum PT, aPTT, and D-dimer values) were evaluated.

Results: The serum CA-125, aPTT, FIB and D-dimer levels were statistically different between OEM patients in the stages I to (and) II group and those in the stages III and IV group $(\mathrm{P}<0.05)$. However, a statistical difference in CA 19-9 levels and TT was only found between patients with stages III and IV OEM. In receiver operating characteristic (ROC) curve analysis of single indexes, the area under the ROC curve values for CA-125, CA19-9, aPTT, TT, FIB, and D-dimer were 0.953, 0.512, 0.66, 0.576, 0.573, and 0.624, respectively, for diagnosing stage III and stage IV OEM. In ROC curve analysis of combined indexes, the AUC values for aPTT + D-dimer, CA-125 + D-dimer, CA-125 + aPTT and CA-125 + D-dimer + aPTT were $0.672,0.954,0.958$, and 0.961 , respectively.

Conclusions: The combined index of CA-125, aPTT, and D-dimer is a valid noninvasive preoperative method for the evaluation of moderate and severe OEM, and may help to decrease the interval between the first complaint and a definitive diagnosis.

Keywords: Cancer antigen-125 (CA-125); cancer antigen 19-9 (CA19-9); prothrombin time (PT); activated
partial thromboplastin time (aPTT); thrombin time (TT); fibrinogen (FIB); D-dimer; endometriosis; noninvasive
evaluation

Submitted Jan 18, 2021. Accepted for publication Apr 01, 2021.

doi: $10.21037 / \mathrm{apm}-21-481$

View this article at: http://dx.doi.org/10.21037/apm-21-481

\section{Introduction}

Endometriosis is a common (prevalent) condition of the female reproductive system, with approximately $5-10 \%$ of reproductive (premenopausal) women suffering from the disorder (1). The main characteristic of endometriosis is the presence of endometrial-like tissue in body parts other than the uterine cavity, typically in the lower abdominal or pelvic areas $(2,3)$. Currently, histologic examinations are considered to be the gold standard for diagnosing 
endometriosis; however, this approach is invasive, as patients must undergo laparoscopy or laparotomy for the obtainment of tissue samples (4). It can take 8 to 10 years to receive a diagnosis of endometriosis after symptom onset (5). Although imaging modalities like transvaginal ultrasound and magnetic resonance imaging (MRI) have improved diagnosis treatment for patients with endometriosis, preoperative assessment still far from ideal because they are operator dependent $(6,7)$, meaning further improvement is needed. Furthermore, severe endometriosis usually causes significant clinical symptoms in patients and makes surgery extremely difficult.

Some serum biomarkers usually change in patients with endometriosis before surgery; however, no biomarkers that can be used to noninvasively diagnose endometriosis with sufficient sensitivity and specificity has yet to be conclusively reported (8). Moreover, no single biomarker has been proven to possess unequivocal clinical usefulness for the diagnosis of endometriosis (9). Thus, ideal biomarkers for the early and noninvasive diagnostic assessment of endometriosis are well worth exploring.

Serum cancer antigen (CA) 125 , a transmembrane glycoprotein, was reported in earlier studies to be elevated in women with endometriosis, and to be valuable in the diagnosis and evaluation of endometriosis (10). However, in some women with endometriosis, serum CA-125 is still within normal range. In another study, serum CA 19-9 was found at significantly higher levels in patients with endometriosis at various stages (11); however, other studies have observed no such association $(12,13)$. A recent study found that patients with endometriosis may display obvious elevation of CA-19-9, the sensitivity of which may be similar to or lower than that of CA-125 (8).

Endometriosis has been traditionally viewed as a hormonal disease. However, Wu et al. recently found that hypercoagulability (14) raises the possibility that these coagulation parameters could be used as biomarkers of endometriosis. Activated partial thromboplastin time (aPTT), thrombin time (TT), fibrinogen (FIB), prothrombin time (PT), and D-dimer level are standard coagulation screening tests in modern clinical practice. In their study, Wu et al. noted that compared to healthy women, patients with ovarian endometriosis (OEM) had considerably shorter aPTT and TT, as well as increased fibrinogen levels; although, for the majority of parameters, the values were within the normal range (14). Meanwhile, another study found TT to have no statistical relationship with endometriosis (15).
From these findings, it can be gathered that some serum biochemical markers may be related to the ectopic formation of endometrial tissue and can change significantly before surgical treatment. Although these serum markers have some clinical value, reports on their utility for assessing the severity of endometriosis have failed to produce a unanimous conclusion. Thus, CA-125, CA 199 , and coagulation and fibrinolysis parameters need to be further studied in patients with endometriosis in order to determine their value as early and noninvasive diagnostic biomarkers for the condition.

Therefore, in the present study, we compared the serum levels of CA-125, CA19-9, aPTT, TT, PT, FIB, and D-dimer between women with and without OEM, analyzed the correlations of these markers with OEM, and further discussed the clinical value of multi-index combinations in the early and noninvasive diagnostic assessment of OEM. We present the following article in accordance with the STARD reporting checklist (available at http://dx.doi. org/10.21037/apm-21-481).

\section{Methods}

\section{Patients and specimens}

A total of 413 reproductive-age patients (mean age $28.98 \pm 3.71$; age range, $21-45)$ with a histologic diagnosis of OEM who were treated in Tianjin Central Hospital of Obstetrics and Gynecology (Tianjin Treatment Center of Endometriosis) between July 2017 and June 2019 were recruited into this study. A control group comprising 158 cycling women without OEM who were matched to the OEM patients by age was also enrolled. All women included in this study were admitted during endometrial hyperplasia and had undergone laparoscopy or laparotomy. Exclusion criteria included patients with OEM who were taking gonadotropin-releasing hormone agonists, progestins, or oral contraceptive pills, and those who were menstruating during operative period. Patients with ruptured endometrial cysts were also excluded, as they show marked increases in serum CA125 and CA19-9 levels (16). None of the subjects were smokers or had used any anticoagulants, steroid hormones, oral contraceptives, antidiabetic drugs, or other medications in the 6 months before the operation.

Patient medical records were comprehensively reviewed, and variables such as age, height $(\mathrm{cm})$, weight $(\mathrm{kg})$, fertility status, gravidity, and International Federation of Gynecology and Obstetrics (FIGO) stage were collected. 
The body mass index (BMI) of each subject was also calculated.

All procedures performed in this study involving human participants were in accordance with the Declaration of Helsinki (as revised in 2013). Ethical approval for this study was granted by the Ethics and Research Committee of the Hospital. All subjects gave their informed consent before participating in the study.

\section{Blood assays}

Biochemical serum analysis of CA-125, CA 19-9, aPTT, TT, PT, D-dimer, and FIB was the focus of the clinical testing. CA-125 is a glycoprotein, the expression of which may be found in fetal epithelial tissue and in the normal epithelia of the fallopian tubes, cervix, or bronchus in adult women. It has particular importance as a marker of serosa membrane carcinoma and undifferentiated ovarian carcinoma (17). CA 19-9 is a pentasaccharide belonging to a class of oncofetal antigens. During the fetal period, CA 19-9 synthesis takes place in the stomach's epithelial structures, while in adults, it is produced at significantly lower levels. Furthermore, it has recently been reported that CA 19-9 may be produced in gallbladder, pancreatic, and bronchial glandular structures, as well as in some tumors of the female reproductive system (8). The aPTT, TT, PT, FIB, and D-dimer are commonly used to evaluate coagulation function, and are convenient, fast, and economical to obtain.

Prior to blood sample collection, the subjects fasted for 12 hours, and all specimens were collected via venipuncture before 9:00 am. The Sysmex CS-5100 system (Sysmex, Kobe, Japan) with Dade Behring reagents (Dade Behring, Marburg, Germany) was used to measure preoperative serum aPTT, TT, PT, D-dimer levels, and FIB levels. A ChemiLuminescence ImmunoAssay System (Roche-e601, Cobas, Switzerland) and the Roche Elecsys kit were used to measure the levels of preoperative serum CA-125 and CA19-9 in the hospital's clinical laboratory. The reference ranges used were as follows: CA-125: 0-35 U/mL; CA 19-9: 0-27 U/mL; aPTT: 22-32 seconds, TT: 15.2-21.2 seconds; PT: $8.3-14.3$ seconds; D-dimer: $0-0.55 \mathrm{mg} / \mathrm{L}$; and FIB: $1.8-3.8 \mathrm{~g} / \mathrm{L}$.

\section{Statistical analysis}

Continuous data were presented as mean \pm standard deviation, and the Kolmogorov-Smirnov test was performed to analyze normally distributed variables. Associations of categorical and continuous variables were analyzed with Student's t-test. For categorical variables with non-normal distribution, the Mann-Whitney $U$ test was applied. Receiver operator characteristic (ROC) curve analysis was performed to determine the cut-off values for aPTT, TT, PT, D-dimer, FIB, CA-125, and CA 19-9. The Youden index (sensitivity + specificity - 1) was also calculated, and the cut-off value with the maximum Youden index was considered to be the optimal cut-off value. The level of statistical significance was set as $\mathrm{P}<0.05$. SPSS 16.0 software for Windows (SPSS Inc., Chicago, IL, USA) was used to perform the statistical analysis.

\section{Results}

The characteristic information of 413 patients with a histologic diagnosis of OEM and 158 controls without OEM is detailed in Table 1. Among the 413 cases in the OEM cohort, 143 women had stage I to II, 139 women had stage III, and 131 women had stage IV disease. Among the control cases, the primary diagnoses were: paraovarian cyst $(n=39)$, simple cyst of the ovary $(n=37)$, corpus luteum cyst of the ovary $(n=23)$, cystadenoma of the ovary $(n=21)$, cervical intraepithelial neoplasia (CIN) III $(\mathrm{n}=18)$, ovarian fibroma $(n=16)$, and mesosalpinx cyst $(n=4)$.

The OEM patients and the controls did not differ significantly in terms of age or BMI (both $\mathrm{P}>0.05$ ). Furthermore, we found no difference in gravidity, delivery, or miscarriage between patients with stage I to II OEM and the control group $(\mathrm{P}>0.05)$; however, OEM patients of stages III and IV had a high rate of infertility and low rates of delivery and miscarriage $(\mathrm{P}<0.05)$.

The levels of serum CA-125, CA 19-9, FIB, and D-dimer, together with the aPTT and TT, were compared between the OEM patients and the control group (Table 2). We observed no difference in CA-125 ( $\mathrm{P}=0.275)$, CA19-9 $(\mathrm{P}=0.232)$, PT $(\mathrm{P}=0.154)$, aPTT $(\mathrm{P}=0.141)$, TT $(\mathrm{P}=0.126)$, FIB $(\mathrm{P}=0.823)$, or $\mathrm{D}$-dimer $(\mathrm{P}=0.555)$ between patients with stage I to II OEM and the control group. However, compared to the stage I to II group, stage III and stage IV patients had significantly elevated levels of $\mathrm{CA}-125(\mathrm{P}=0.000$ and $\mathrm{P}=0.000$, respectively), $\mathrm{CA1}-9$-9 $(\mathrm{P}=0.000), \mathrm{FIB}(\mathrm{P}=0.023$ and $\mathrm{P}=0.029$, respectively), and $\mathrm{D}$-dimer $(\mathrm{P}=0.034$ and $\mathrm{P}=0.000$, respectively), as well as a shortened aPTT $(\mathrm{P}=0.022$ and $\mathrm{P}=0.000$, respectively) and TT $(\mathrm{P}=0.023)$. No significant differences were observed in $\mathrm{PT}(\mathrm{P}>0.05)$ among the groups, or in $\mathrm{CA19-9}(\mathrm{P}=0.263)$ or TT $(\mathrm{P}=0.079)$ between 
Table 1 Characteristics of the patients with OEM and the controls

\begin{tabular}{|c|c|c|c|c|}
\hline Characteristics & OEM stage I to II $(n=143)$ & OEM stage III $(n=139)$ & OEM stage IV ( $n=131)$ & Controls $(n=158)$ \\
\hline $\mathrm{P}$ & $0.937^{\mathrm{a}}$ & $0.234^{\mathrm{b}}$ & $0.886^{c}$ & $0.106^{\mathrm{d}}$ \\
\hline BMI $\left(\mathrm{kg} / \mathrm{m}^{2}\right)$ & $21.28 \pm 1.22$ & $21.32 \pm 1.27$ & $21.32 \pm 1.27$ & $21.14 \pm 0.89$ \\
\hline $\mathrm{P}$ & $0.233^{a}$ & $0.789^{b}$ & $0.814^{c}$ & $0.950^{d}$ \\
\hline \multicolumn{5}{|l|}{ Gravidity } \\
\hline 0 & $29(20.28)$ & $61(43.89)$ & 52 (39.69) & $28(17.72)$ \\
\hline 1 & $69(48.25)$ & $30(21.58)$ & $71(54.20)$ & $66(41.77)$ \\
\hline$\geq 2$ & $45(31.47)$ & $48(34.53)$ & $8(6.11)$ & $64(40.51)$ \\
\hline 0 & $47(32.87)$ & $57(41.01)$ & $70(53.44)$ & $47(29.75)$ \\
\hline 1 & $80(55.94)$ & $77(55.40)$ & $59(45.04)$ & $98(62.03)$ \\
\hline$\geq 2$ & $16(11.19)$ & $5(3.60)$ & $2(1.53)$ & $13(8.23)$ \\
\hline $\mathrm{P}$ & $0.584^{\mathrm{a}}$ & $0.042^{b *}$ & $0.000^{c *}$ & $0.032^{d *}$ \\
\hline \multicolumn{5}{|l|}{ Miscarriage } \\
\hline 0 & $74(51.75)$ & $85(61.15)$ & $97(74.05)$ & $70(44.30)$ \\
\hline 1 & $50(34.97)$ & 48 (34.53) & 31 (23.66) & $70(44.30)$ \\
\hline$\geq 2$ & $19(13.29)$ & $6(4.32)$ & $3(2.29)$ & $18(11.39)$ \\
\hline
\end{tabular}

${ }^{a}$, E stage I+II vs. Controls; ${ }^{b}$, E stage I+II vs. E stage III; ${ }^{\text {, }}$ E stage I+II vs. E stage IV; ${ }^{d}$, E stage III vs. E stage IV. Statistically significant P values are indicated with *. OEM, ovarian endometriosis; BMI, body mass index.

stage III patients and the stage I to II group. Compared to the stage I to II group and the control group, patients with stage III and IV OEM had statistically significant elevation of CA-125, CA19-9, FIB, and D-dimer $(\mathrm{P}=0.000$, $\mathrm{P}=0.001, \mathrm{P}=0.000$, and $\mathrm{P}=0.000$, respectively), and a shorter aPTT and TT $(\mathrm{P}=0.000$ and $\mathrm{P}=0.043$, respectively), while no statistical difference was found between the groups in regard to PT.

The above-mentioned parameters were compared between the groups, and CA-125, aPTT, TT, FIB, and D-dimer were subsequently selected for ROC curve analysis with single and combined indicators in order to further study their predictive value for stage III and stage IV OEM.

The area under the curve (AUC) values for CA-125, CA19-9, aPTT, TT, FIB and D-dimer was 0.953, 0.512, $0.66,0.576,0.573$ and 0.624 in the analysis of ROC curve with single index, respectively (Figure $1 A$ ). Because the AUC of CA19-9, TT or FIB was less than 0.6, the CA125, aPTT and D-dimer were chosen for combined-index analysis. The AUC of aPTT + D-dimer, CA-125 + D-dimer, CA-125 + aPTT and CA-125 + D-dimer + aPTT was 0.672, 0.954, 0.958 and 0.961 , respectively. So combined the CA125 , D-dimer and aPTT analysis was the best assessment method for the OEM stage III and stage IV disease, with a sensitivity of $93.8 \%$ and a specificity of $84.7 \%$ (Figure $1 B$ ).

\section{Discussion}

Women with OEM are usually observed to have elevated serum CA-125 and CA19-9 levels, and coagulation parameters. However, at present, endometriosis is diagnosed by postoperative histopathological examination, 
Table 2 Blood markers and coagulation parameters of the patients with OEM and the controls.

\begin{tabular}{|c|c|c|c|c|}
\hline Variable & OEM stage I to II $(n=143)$ & OEM stage III (n=139) & OEM stage IV (n=131) & Controls $(n=158)$ \\
\hline $\mathrm{P}$ & $0.000^{a *}$ & $0.000^{\mathrm{b} *}$ & $0.000^{c *}$ & $0.275^{d}$ \\
\hline CA19-9 (U/L) & $13.10 \pm 8.17$ & $14.52 \pm 12.61$ & $19.59 \pm 17.01$ & $14.17 \pm 7.27$ \\
\hline $\mathrm{P}$ & $0.263^{\mathrm{a}}$ & $0.000^{\mathrm{b} *}$ & $0.001^{c *}$ & $0.232^{d}$ \\
\hline$P$ & $0.274^{\mathrm{a}}$ & $0.622^{b}$ & $0.103^{c}$ & $0.154^{d}$ \\
\hline aPTा (s) & $27.34 \pm 2.90$ & $26.57 \pm 2.67$ & $25.83 \pm 2.54$ & $27.76 \pm 1.79$ \\
\hline $\mathrm{P}$ & $0.022^{a *}$ & $0.000^{\mathrm{b} *}$ & $0.000^{c *}$ & $0.141^{d}$ \\
\hline$\pi \mathrm{T}(\mathrm{s})$ & $18.45 \pm 0.87$ & $18.29 \pm 0.66$ & $18.21 \pm 0.85$ & $18.31 \pm 0.82$ \\
\hline $\mathrm{P}$ & $0.023^{a *}$ & $0.029^{b *}$ & $0.000^{c *}$ & $0.823^{d}$ \\
\hline D-dimer (mg/L) & $0.12 \pm 0.05$ & $0.13 \pm 0.04$ & $0.14 \pm 0.03$ & $0.12 \pm 0.04$ \\
\hline$P$ & $0.034^{a \star}$ & $0.000^{\mathrm{b} *}$ & $0.000^{\mathrm{c} *}$ & $0.555^{d}$ \\
\hline
\end{tabular}

${ }^{a}$, E stage I+I vs. E stage III; ${ }^{b}$, E stage I+II vs. E stage IV; ${ }^{\circ}$, E stage I+II+ Controls vs. E stage III+IV; ${ }^{d}$, E stage I+II vs. Controls. Statistically significant $P$ values are indicated with *. aPTT, activated partial thromboplastin time; TT, thrombin time; FIB, fibrinogen; D-D, D-dimers.

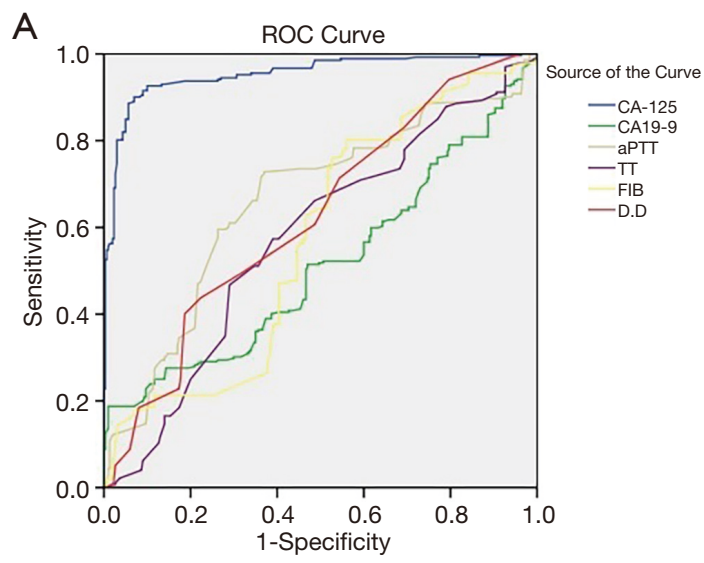

Diagonal segments are produced by ties.

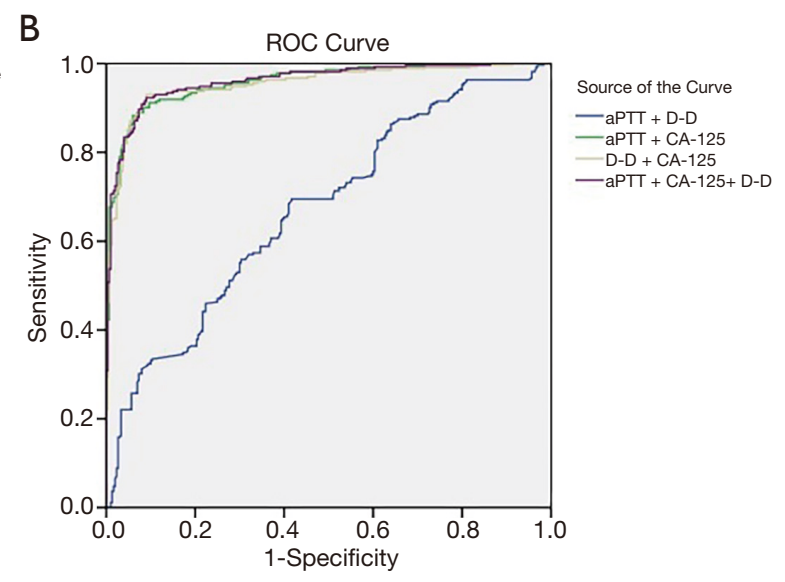

Diagonal segments are produced by ties.

Figure 1 ROC curve analysis of: (A) single indicators (CA-125, aPTT, TT, FIB, and D-D); (B) combined indicators (aPTT+D-D, aPTT+CA-125, CA-125+D-D, and CA-125+D-D+aPTT). ROC, receiver operating characteristic; CA, cancer antigen; aPTT, activated partial thromboplastin time; TT, thrombin time; FIB, fibrinogen; D-D, D-dimers.

because no noninvasively obtainable diagnostic biomarker has been validated for the condition. A delayed diagnosis of endometriosis can cause severe pain for the patient and brings great difficulty to treatment. In our current research, we measured the serum CA-125, CA 19-9, FIB, and D-dimer levels, along with the PT, aPTT, and TT, in women with OEM and in a control group of non-OEM patients. These parameters showed no difference between 
patients with stage I to II OEM and the control group; however, the serum levels of CA-125, FIB, and D-dimer, and the aPTT were statistically different between OEM patients in the stage I to II group and those with stages III and IV disease. Meanwhile, statistical differences in CA 19-9 levels and TT were only observed between patients with stage III and stage IV OEM. For patients with severe OEM (stages III and IV), ROC curve analysis showed that serum CA-125, aPTT, and D-dimer were the best indicators for the evaluation of OEM, and the other 3 indicators were abandoned.

Numerous investigations have been carried out to determine the feasibility of using serum CA-125 to diagnose patients who are clinically suspected as having endometriosis. However, their findings have been inconsistent, primarily in regard to the sensitivity of serum CA-125 $(17,18)$. The challenge in using serum CA-125 as a biomarker of endometriosis can be found in establishing an appropriate cut-off value, because the current value $(35 \mathrm{IU} / \mathrm{mL})$ is the reference for epithelial ovarian cancer. Fluctuations in CA-125 levels have been reported at various points in the menstrual cycle, and elevated CA-125 is a result of especially during menstruation and the rupture of the endometriosis cyst $(16,19)$. Therefore, in the present study, venous blood was drawn from all patients between menstrual periods, and patients who were menstruating and those who had cyst rupture were excluded. Our data indicated that patients with OEM stages III and IV had a significantly elevated CA-125 level compared to patients with OEM stages I to II. CA-125 also had a considerably higher value for predicting severe endometriosis. For severe OEM (stages III and IV), the ROC analysis showed that the cut-off value of serum CA-125 was $22.41 \mathrm{U} / \mathrm{mL}$ (significantly lower than the $35 \mathrm{IU} / \mathrm{mL}$ used as the cutoff for epithelial ovarian cancer), which had a sensitivity and specificity of $90.1 \%$ and $93.0 \%$, respectively. Thus, serum CA-125 is a satisfactory diagnostic marker for OEM, and severe OEM can be suspected when the CA125 level is high $(\geq 22.41 \mathrm{U} / \mathrm{mL})$ and there are obvious clinical symptoms and ultrasonic signs. However, in our study, statistical differences in CA 19-9 levels were only observed between OEM patients in the stage I and II group and those in the stage IV group. Furthermore, the ROC analysis showed that the serum CA19-9 had no diagnostic significance $(\mathrm{P}=0.682)$. Therefore, we consider $\mathrm{CA19}-9$ to exhibit low sensitivity for the diagnosis of OEM and to be inappropriate for use in diagnostic evaluation.

A hypercoagulable state is also thought to be present in moderate and severe endometriosis (14), and these coagulation parameters (aPTT, PT, TT, FIB and D-dimer) could serve as biomarkers for noninvasive diagnosis of endometriosis. APTT is the most common routine coagulation test performed in laboratories. Emerging evidence suggests that a short aPTT is associated with hypercoagulability (20). We found that women with OEM stages III and IV had a short aPTT, although it was still in the normal range. Further, the level of aPTT was not different between women with mild OEM (stage I to II) and those without OEM. The ROC analysis showed that the serum aPTT was a plausible noninvasive diagnostic biomarker for severe OEM. APTT is often used in combination with PT for evaluation of the speed at which blood clotting occurs, and it is not used alone. However, in the present study, serum PT showed no statistical difference between patients with OEM and the control group. Our data failed to indicate that PT could be used as a marker for diagnosing OEM, and further study is required to investigate this matter.

The fibrinolytic system has a close relationship with the coagulation cascade and has been described as having involvement in the development of endometriosis (21). Furthermore, the TT reflects fibrinogen-fibrin conversion and is now considered to represent a procoagulant milieu (22). The relationships of FIB, D-dimer, and TT with endometriosis are not well reported. Our data indicated that the serum FIB and D-dimer levels were statistically elevated in OEM patients of stages III and IV. However, further ROC analysis showed that the serum D-dimer, but not FIB, could be used for the evaluation of severe OEM. Meanwhile, a statistical difference in serum TT was only observed between the stage I to II OEM group and the stage IV OEM group. We were also unable to confirm alterations in coagulation variables in women with OEM (15).

The concentrations of the serum parameters used in the present study were mostly within the normal range in women with OEM, which creates some questions regarding the clinical relevance of our findings. However, when there are several different measurements that are differentially distributed in normal and diseased populations, these serum parameters could potentially be used as biomarkers. Taking our results of elevated levels of FIB and D-dimer, and shortened aPTT and TT in the peripheral blood plasma of patients with severe OEM, the evidence of a hypercoagulable state was unmistakable. The combination of CA-125, D-dimer, and aPTT produced a sensitivity 
of $93.8 \%$ and a specificity of $84.7 \%$ for the noninvasive preoperative evaluation of endometriosis, which were higher than those reported by Vodolazkaia (23).

Our findings may help to reduce the interval between the first complaint by a patient and a definitive diagnosis of moderate (stages III) or severe OEM (stages IV). The larger-sample multicenter studies should be conducted to further explore the validation of biomarkers for noninvasive evaluation of endometriosis. Also, current reference ranges for tumor markers and coagulation markers are not suitable for endometriosis. Determination of reference ranges of blood parameters for endometriosis by a larger sample study would aid in the clinical evaluation and scientific research of endometriosis.

\section{Acknowledgments}

Funding: None.

\section{Footnote}

Reporting Checklist: The authors have completed the STARD reporting checklist. Available at http://dx.doi.org/10.21037/ apm-21-481

Data Sharing Statement: Available at http://dx.doi. org/10.21037/apm-21-481

Conflicts of Interest: Both authors have completed the ICMJE uniform disclosure form (available at http://dx.doi. org/10.21037/apm-21-481). The authors have no conflicts of interest to declare.

Ethical Statement: The authors are accountable for all aspects of the work in ensuring that questions related to the accuracy or integrity of any part of the work are appropriately investigated and resolved. All procedures performed in this study involving human participants were in accordance with the Declaration of Helsinki (as revised in 2013). Ethical approval for this study was granted by the Ethics and Research Committee of the Hospital. All subjects gave their informed consent before participating in the study.

Open Access Statement: This is an Open Access article distributed in accordance with the Creative Commons Attribution-NonCommercial-NoDerivs 4.0 International License (CC BY-NC-ND 4.0), which permits the non- commercial replication and distribution of the article with the strict proviso that no changes or edits are made and the original work is properly cited (including links to both the formal publication through the relevant DOI and the license). See: https://creativecommons.org/licenses/by-nc-nd/4.0/.

\section{References}

1. Giudice LC, Kao LC. Endometriosis. Lancet 2004;364:1789-99.

2. Cloke B, Christian M. The role of androgens and the androgen receptor in cycling endometrium. Mol Cell Endocrinol 2012;358:166-75.

3. Simitsidellis I, Saunders PT, Gibson DA. Androgens and endometrium:new insights and new targets. Mol Cell Endocrinol 2018;465:48-60.

4. de Ziegler D, Borghese B, Chapron C. Endometriosis and infertility: pathophysiology and management. Lancet 2010;376:730-8.

5. Ahn SH, Singh V, Tayade C. Biomarkers in endometriosis: challenges and opportunities. Fertil Steril 2017;107:523-32.

6. Abrao MS, da C Goncalves MO, Dias JA Jr, et al. Comparison between clinical examination, transvaginal sonography andmagnetic resonance imaging for the diagnosis of deep endometriosis. Human Reproduction 2007;22:3092-7.

7. Chami'e LP, Blasbalg R, Ricar-do Mendes AP, et al. Findings of pelvic endo-metriosis at transvaginal US, MR imaging, and laparoscopy. Radiographics 2011;31:E77-100.

8. Fassbender A, Burney RO, Dorien FO, et al. Update on Biomarkers for the Detection of Endometriosis. BioMed Res Int 2015;2015:130854.

9. Nisenblat V, Bossuyt PM, Shaikh R, et al. Blood biomarkers for the non-invasive diagnosis of endometriosis. Cochrane Database Syst Rev 2016;(5):CD012179.

10. Barbieri RL, Niloff JM, Bast RC Jr, et al. Elevated serum concentrations of CA-125 in patients with advanced endometriosis. Fertil Steril 1986;45:630-4.

11. Harada T, Kubota T, Aso T. Usefulness of CA19-9 versus CA125 for the diagnosis of endometriosis. Fertil Steril 2002;78:733-9.

12. Somigliana E, Vigano P, Tirelli AS, et al. Use of the concomitant serum dosage of CA 125, CA 19-9 and interleukin-6 to detect the presence of endometriosis. Results from a series of reproductive age women undergoing laparoscopic surgery for benign gynaecological 
conditions. Hum Reprod 2004;19:1871-6.

13. Xavier P, Beires J, Belo L, et al. Are we employing the most effective CA 125 and CA 19-9 cut-off values to detect endometriosis? Eur J Obstet Gynecol Reprod Biol 2005;123:254-5.

14. Wu Q, Ding D, Liu X, et al. Evidence for a hypercoagulable state in women with ovarian endometriomas. Reprod Sci 2015;22:1107-14.

15. Viganò P, Ottolina J, Sarais V, et al. Coagulation Status in Women With Endometriosis. Reprod Sci 2018;25:559-65.

16. Kurata H, Sasaki M, Kase H, et al. Elevated serum CA125 and CA19-9 due to the spontaneous rupture of ovarian endometrioma. Eur J Obstet Gynecol Reprod Biol 2002;105:75-6.

17. Hirsch M, Duffy JM, Deguara CS, et al. Diagnostic accuracy of Cancer Antigen 125 (CA125) for endometriosis in symptomatic women: a multicenter study. Eur J Obstet Gynecol Reprod Biol 2017;210:102-7.

18. Karimi-Zarchi M, Dehshiri-Zadeh N, Sekhavat L, et al. Correlation of CA-125 serum level and clinicopathological characteristic of patients with endometriosis. Int J Reprod Biomed 2016;14:713-8.

Cite this article as: Zhao K, Qu P. Noninvasive evaluation of ovarian endometriosis: a single-center experience. Ann Palliat Med 2021;10(4):4728-4735. doi: 10.21037/apm-21-481
19. Kafali H, Artuc H, Demir N. Use of CA125 fluctuation during the menstrual cycle as a tool in the clinical diagnosis of endometriosis; a preliminary report. Eur J Obstet Gynecol Reprod Biol 2004;116:85-8.

20. Korte W, Clarke S, Lefkowitz JB. Short activated partial thromboplastin times are related to increased thrombin generation and an increased risk for thromboembolism. Am J Clin Pathol 2000;113:123-7.

21. Gilabert-Estelles J, Castello R, Gilabert J, et al. Plasminogen activators and plasminogen activator inhibitors in endometriosis. Front Biosci 2005;10:1162-76.

22. Mina A, Favaloro EJ, Koutts J. Relationship between short activated partial thromboplastin times, thrombin generation, procoagulant factors and procoagulant phospholipid activity. Blood Coagul Fibrinolysis 2012;23:203-7.

23. Vodolazkaia A, El-Aalamat Y, Popovic D, et al. Evaluation of a panel of 28 biomarkers for the non-invasive diagnosis of endometriosis. Hum Reprod 2012;27:2698-711.

(English Language Editor: J. Reynolds) 